\title{
CONCLUSION : ENTRE MARCHÉ, ÉTAT ET SOCIÉTÉ CIVILE: EXPLORATION DE LA NATURE HYBRIDE ET DES PHÉNOMÈNES D'HYBRIDATION DES ORGANISATIONS
}

\author{
Sophie Del FA ${ }^{1}$, François Lambotte ${ }^{2}$, Consuelo VÁSQUEZ ${ }^{3}$
}

Constatant que les recherches sur l'hybridité organisationnelle résolvent de façon trop souvent binaire les tensions inhérentes à ces formes, ce numéro a pour ambition de mettre en lumière les paradoxes et les ambiguités hybrides comme des dynamiques qui émergent de l'organisation elle-même et qui, in fine, la constituent. En partant des phénomènes et évènements communicationnels (interactions, discours, pratiques discursives), les neuf articles de ce dossier questionnent les processus d'hybridation organisationnelle du point de vue (1) des finalités et des identités organisationnelles, mais aussi individuelles et des imaginaires collectifs; (2) des logiques multiples qui les traversent : professionnelles, sociales, artistiques, financières, par exemple et (3) des spécificités des actrices et des acteurs qui les animent. Ces articles soulignent la nécessité d'explorer les modalités d'expression, les traces et les pratiques des processus d'hybridation organisationnelle et des tensions qu'ils génèrent. Les travaux présentés démontrent la valeur théorique et conceptuelle d'une approche communicationnelle

1 Sophie Del Fa est professeure en communication au département des Arts et Lettres à l'Université du Québec à Chicoutimi.

2 François Lambotte est professeur à l'École de communication de l'Université catholique de Louvain (Belgique).

3 Consuelo Vásquez est professeure au département de Communication sociale et publique de l'UQAM et co-fondatrice du Groupe de Recherche sur la Communication Organisante (ReCOr).

Recherches en communication, $\mathrm{n}^{\circ} 47$ - Article publié le 26/09/2019 
comme lentille permettant d'interroger ces processus travaillés « de l'intérieur».

Le premier article de ce dossier thématique, intitulé "L'hybridité : une composante ancienne, mais aujourd'hui démultipliée de la communication publique " amorce la réflexion sur le caractère hybride de l'organisation en se penchant plus particulièrement sur le champ de la communication publique. L'auteur, Dominique Bessière, y explore l'entrecroisement des modèles organisationnels publics, privés et politiques, qui dans les dernières décennies, ont transformé le secteur public, et conséquemment, la communication publique. L'hybridité se retrouve d'une part dans la bureaucratisation des institutions publiques dans laquelle cohabitent le public et le politique. D'autre part, la communication publique, comme profession articule des fonctions juridiques, propres des institutions d'état, avec l'administration et le management. Ajoutons à cela, la professionnalisation du champ dans les années 80 et sa normalisation inspirée des modèles privés, reposant sur l'efficacité et la performance. Il en résulte, selon l'auteur, une communication complexe par l'hybridité institutionnelle qui la conditionne entre politique et public, mais en référence croissante au modèle d'efficacité privé.

Dans leur article « L'hybridation discursive dans la communication sur la responsabilité sociétale des entreprises : le cas des banques « engagées » " Andrea Catellani et Amaia Errecart explorent le discours institutionnel du Crédit coopératif et de la banque Triodos. Toutes deux représentantes et pionnières du mouvement de la " finance éthique », ces banques participent au phénomène de la « responsabilité sociétale d'entreprise » (RSE). Une analyse sémio-discursive est développée pour comprendre comment le discours de ces banques construit des formes de gestion rhétorique de la coprésence de différentes valeurs, valorisations et logiques. Par une analyse fine, les auteurs concluent que l'ensemble du discours des deux banques est en même temps une expression et un facteur de leur identité hybride, de par la performativité 
propre des discours composites fondés sur un compromis entre les mondes marchand et civique.

Valérie Larroche et Valentyna Dymytrova réalisent, pour leur part, une immersion originale 'dans' l'hybridité définie comme une articulation entre plusieurs logiques, objectifs, croyances et valeurs de différentes et différents actrices et acteurs, à partir de leur participation active à un projet de recherche multipartenaire, qui vise la conception d'une boîte à outils facilitant la réutilisation des données ouvertes. L'article intitulé « Les dynamiques d'hybridité au sein d'un projet multipartenaire " mobilise l'approche constitutive de la communication organisationnelle pour étudier l'agentivité des textes, des objets techniques ainsi que les jeux des actrices et acteurs en tant que modalités importantes d'hybridité, et ce dans les situations de médiation où l'articulation des cadres de fonctionnement et des cadres d'usage est à l'œuvre.

Sophie Huys rend compte de son implication à Greenpeace Belgique dans le but de saisir les tensions entre la logique de marché et la mission non lucrative vécues par les professionnels autour d'une plateforme numérique collaborative Greenwire.org. Vues comme étant caractérisée par une pluralité d'idéaux types, de cultures, de mécanismes de coordination, de rationalités, et de logiques d'action, cette ONG, parangon de l'organisation hybride, est mue par des tensions et des contradictions. En analysant les interactions entre les actrices et acteurs participants à cette plateforme, l'auteure révèle plusieurs enjeux, moteur des tensions comme la professionnalisation des membres, le conflit entre le choix de l'action directe ou de l'action stratégique ou encore entre l'autonomie et le contrôle de la plateforme. Il en résulte une étude approfondie et originale d'une organisation à but non lucratif qui en révèle les complexités à travers l'analyse d'un outil numérique.

Dans l'article intitulé « Organisations émergentes du monde de l'art : une analyse de l'hybridité des logiques d'action, » Guillaume Sirois et Guy Bellavance explorent le caractère hybride de l'organisation dans le monde de l'art. En se basant sur cinq études 
de cas réalisées auprès d'organisations artistiques émergentes, les auteurs mettent en dialogue trois logiques qui structurent les organisations étudiées, soit la logique créative, entrepreneuriale et sociale. Chacune de ces logiques répond à des systèmes de valeurs différents qui, dans les cas étudiés, renvoient à la valeur de l'art. L'article montre avec finesse la manière dont les responsables de ces organisations émergentes jonglent avec ces logiques, sublimant les tensions qui naissent de cette « axiologie hybride » dans laquelle coexistent des objectifs stratégiques, des formes d'engagement et des marqueurs de succès différenciés. Les auteurs soulèvent une conséquence inespérée, mais toutefois significative de cette hybridation : la difficulté pour ces organisations d'être reconnues par les institutions culturelles qui, entre autres, subventionnent ces initiatives.

Continuant dans le monde de l'art, Joanne Jojczyk, dans l'article intitulé « Création artistique et participation : l'émergence d'une vision partagée et d'une esthétique hybride de l'œuvre, » aborde l'hybridation organisationnelle en s'interrogeant sur la nature du projet artistique participatif. Plus particulièrement, elle questionne " comment des choix esthétiques sont négociés dans un processus de création participatif par des acteurs projetant des imaginaires multiples ? \C'est la notion d'imaginaire qui lui permet de rendre compte de l'ADN particulier de ce type de projet dans lequel, selon l'auteure, « l'hybridité est ancrée de facto » à travers la cohabitation des voix et des imaginaires des multiples participants au projet. À partir d'une étude ethnographique du Grand Huit, projet artistique et participatif inscrit dans les festivités de la capitale européenne Mons 2015, Jojczyk montre comment les imaginaires artistiques, gestionnaires et territoriaux créent une esthétique hybride et partagée. L'entente commune construite dans et par les interactions devient un script qui facilite et contraint les actions des participants.

De leur côté, Sandrine Roginsky et Christel Christophe proposent d' « étudier l'hybridation organisationnelle par le prisme des publics : les publics comme pierre angulaire des tensions qui animent une association de solidarité internationale ». Sujet 
rarement abordé sous cet angle, les auteures questionnent la manière dont les publics figurés dans les discours des membres $\mathrm{du}$ personnel d'une association active dans la coopération au développement participent à l'émergence de tensions qui constituent l'organisation. En particulier, les auteures s'intéressent à la performativité des publics figurés et à la façon dont agissent les membres de l'organisation dans leur pratique de mobilisation et de sensibilisation des publics à leur projet. Les auteures mettent en évidence trois manières de figurer les publics : en convoquant leur propre expérience, en faisant référence à des échanges ou expériences collectives ou en les figurant sous forme de chiffres. Ces figures, parce qu'elles sont elles-mêmes porteuses de valeurs de visions variables, peuvent lorsqu'elles sont convoquées produire les tensions organisantes au cœur du processus d'hybridation.

Dans leur article intitulé "Communication et dynamiques d'hybridation dans les organisations de type laboratoire vivant : un regard constitutif sur le démarrage d'un living lab au service des aînés ", Dany Baillargeon et Hélène Bruneau analysent le rôle constitutif des tensions associées aux activités fondamentales de l'organisation que sont, selon la typologie de Smith et Lewis, l'apprentissage, l'appartenance, l'accomplissement et l'organizing. À l'instar de Roginsky et Christophe, Baillargeon et Bruneau analysent les figures ventriloquées par les actrices et acteurs lors des réunions de démarrage du projet de laboratoire vivant. L'analyse conversationnelle proposée révèle les figures contradictoires présentifiées lors des discussions liées à la mission d'un laboratoire à la croisée de la recherche et du service à la société, à la conception de ce qu'est le vieillissement ou encore sur la gouvernance et les finalités du laboratoire en termes de recherche, d'enseignement ou de lucres. Les auteurs considèrent que ces diverses contradictions se rapportent principalement à une tension entre l'attachement à des figures traditionnelles du chercheur et celles en devenir dans le laboratoire innovant. Ainsi, les auteurs posent le détachement à des figures anciennes et le rattachement à certaines figures nouvelles comme deux modalités d'hybridation. 
Christelle Sukadi Mangwa, dans son article intitulé « Analyse d'une organisation hybride par l'approche de Thomas et Hardy : le cas de la mise en place d'une Activité Génératrice de Revenus dans une ONG de développement en République Démocratique du Congo " propose, comme son titre l'indique, une analyse critique et longitudinale des discours issus de la mise en place d'une Activité Génératrice de Revenus. Elle aborde son étude en vue d'appréhender les tensions entre les logiques institutionnelles qui caractérisent l'hybridité de l'ONG. En somme, Sukadi Mangwa met en évidence la tension entre une logique sociale et une logique financière dans la mise en place de cette activité. Cela permet à l'auteure de voir l'hybridité non pas seulement à travers les tensions, mais aussi, et surtout à travers les jeux de pouvoir. L'hybridité est conçue comme un lieu d'affrontements discursifs dans lequel chacun veut imposer sa vision.

Pour conclure, nous espérons que ce numéro suscitera de nouvelles avenues de recherches pour dépasser une vision binaire du phénomène d'hybridation organisationnelle et de le considérer plutôt dans et par les tensions qui le constituent. Il nous semble que cela répond aussi à la nécessité urgente de comprendre de manière plus fine les mécanismes à l'œuvre dans la société contemporaine. En effet, nous assistons à un double mouvement (en apparence contradictoire) de dislocation et de recomposition des organisations.

D'une part, les organisations dites « traditionnelles » qui structurent historiquement les sociétés du «nord» se disloquent. En outre, les grandes industries se font de plus en plus rares étant délocalisées dans les pays " du sud "; de même que les sièges sociaux des entreprises, qui préfèrent s'installer dans des pays aux politiques fiscales plus légères. De plus, les gouvernements libéraux-socialistes et néolibéraux, délèguent ou sous-traitent certaines de ses missions « publiques » au secteur privé. On peut penser également à la dislocation des partis politiques et à celles des organisations syndicales qui perdent de leur légitimité tant auprès des travailleuses et travailleurs qui se sentent abandonné- 
e-s que des organisations patronales qui ne se sentent plus liées par une concertation sociale pour atteindre leurs objectifs financiers.

D'autre part, en parallèle à ce mouvement de décomposition/ dislocation des archétypes organisationnels, on voit apparaitre des phénomènes que l'on désigne comme des recompositions organisationnelles qui sont elles aussi particulièrement éclatées. Par exemple, surfant sur le modèle néolibéral, on peut citer l'autoentrepreneuriat (start-up nation) et l'essor de l'économie disruptive/collaborative représentée par Uber, AirBnB, ferme du clique, etc. En même temps sont valorisés des modèles dits « alternatifs » favorisant une économie circulaire et des stratégies de gestions coopératives (pensons au Bâtiment 7 à Montréal). Dans une direction de recomposition en vue de "révolution ", des mouvements citoyens de grande ampleur s'organisent dans plusieurs endroits du monde (les gilets jaunes bien sûr, mais aussi les soutiens aux migrantes et migrants du parc Maximilien en Belgique, les Marches pour le climat portées par les étudiantes et étudiants, les manifestations en Algérie, etc.). Tout cela s'accompagnant de l'hyperpuissance difficile à freiner des entreprises œuvrant dans les technologies numériques et l'intelligence artificielle (les GAFAM pour ne nommer qu'elles).

Bref, les organisations puisent dans différentes logiques économiques et politiques tendant alors soit à renforcer le système existant (le néolibéralisme) soit à y résister soit en l'hybridant considérablement et en créant alors des variations organisationnelles nouvelles. Il est donc primordial de se pencher sur les mécanismes, les pratiques, les discours, les perceptions médiatiques et individuelles de cette multiplication des « façons de s'organiser $»$.

Enfin, nous tenons à souligner une des limites des travaux présentés dans ce numéro : ils s'inscrivent principalement au sein des épistémologies dites du Nord sans les remettre en question. Pourtant, il est temps de dépasser une vision occidentalisée de l'organisation, comme l'esquisse d'ailleurs Sukadi Mangwa en se 
penchant sur le cas de la République Démocratique du Congo et ce, en ouvrant l'approche communicationnelle vers des épistémologies non occidentales (épistémologies dites du « sud » et écologies de savoirs), qui peuvent enrichir et surtout décloisonner la recherche en communication. Étaient également absentes les approches féministes qui permettraient aussi d'approcher les organisations sous un angle non hégémonique et sensible aux minorités et aux 'subalternes'. En somme, ce numéro invite à concevoir la complexité organisationnelle contemporaine tout en soulignant les écueils dans lesquels il faudra éviter de tomber à l'avenir.

\footnotetext{
(c) (1) $(9)$
} «Attribution - Pas d'Utilisation Commerciale - Pas de Modification 4.0 International» (CC BY-NC-ND) 